ISSN 1112-9867

http://www.jfas.info

\title{
ENHANCED INDEX TRACKING IN PORTFOLIO OPTIMIZATION WITH TWO-STAGE MIXED INTEGER PROGRAMMING MODEL
}

\author{
W. S. Lam ${ }^{1, *}$, S. H. Jaaman ${ }^{2}$ and W. H. Lam ${ }^{1}$ \\ ${ }^{1}$ Centre for Mathematical Sciences, Centre for Business and Management, Department of \\ Physical and Mathematical Science, Faculty of Science, Universiti Tunku Abdul Rahman, \\ 31900 Kampar, Perak, Malaysia \\ ${ }^{2}$ School of Mathematical Sciences, Faculty of Science and Technology, Universiti \\ Kebangsaan Malaysia, 43600, Bangi, Selangor, Malaysia
}

Published online: 17 October 2017

\begin{abstract}
Enhanced index tracking is a portfolio management which aims to construct the optimal portfolio to generate higher return than the benchmark index return at minimum tracking error without purchasing all the stocks that make up the index. The objective of this paper is to propose a two-stage mixed integer programming model to improve the existing single-stage mixed integer programming model for tracking FBMKLCI Index in Malaysia. The optimal portfolio performance of both models are determined and compared in terms of portfolio mean return, tracking error, excess return and information ratio. The results of this study indicate that the optimal portfolio of the proposed model generates weekly excess return over the benchmark FBMKLCI index return at minimum tracking error. Besides that, the proposed model is able to outperform the existing model in tracking the benchmark index.
\end{abstract}

Keywords: mean return; tracking error; optimal portfolio; portfolio performance.

Author Correspondence, e-mail: lawms@utar.edu.my

doi: http://dx.doi.org/10.4314/jfas.v9i5s.1 


\section{INTRODUCTION}

Index tracking is a popular type of portfolio management in stock market investment [1]. In index tracking, the fund managers and investors aim to construct the optimal portfolio to generate similar return with the benchmark index return without purchasing all the stocks that make up the index [2]. Tracking error is a risk measure of how closely the optimal portfolio return follows the benchmark index return [3]. In index tracking, the portfolio tracks the benchmark index perfectly if there is zero tracking error. The mixed integer programming model was introduced to construct the optimal portfolio for index tracking problem by minimizing the tracking error [2]. Different index tracking models have been developed and studied by the past researchers in tracking different stock market index [4-9].

Enhanced index tracking is an improved portfolio management which focuses on generating higher portfolio mean return than the benchmark index return besides minimizing the tracking error [10]. Tracking error and excess return of the portfolio over the benchmark index are two main elements in enhanced index tracking [11-12]. The objective of this paper is to propose a two-stage mixed integer programming model to improve the existing single-stage mixed integer programming model $[2,10]$ for enhanced index tracking problem. The proposed model aims to outperform the existing model by generating higher portfolio mean return in tracking the benchmark index in Malaysia. The optimal portfolio performance of both models are determined and compared in terms of portfolio mean return, tracking error, excess return and information ratio. The rest of the paper is organized as follows. The next section describes the materials and methods used in this study. Section 3 discusses about the empirical results of the study. Section 4 concludes the paper.

\section{MATERIAL AND METHOD}

\subsection{Material}

FTSE Bursa Malaysia Kuala Lumpur Composite Index (FBMKLCI) is the leading indicator of the performance of the Malaysia stock market and economy that consists of 30 stocks listed on the Malaysian Main Market [13]. In this study, the data consists of weekly return of stocks in FBMKLCI Index from January 2012 until December 2015. This data is applied in the existing single-stage mixed integer programming model (index tracking) and the proposed 
two-stage mixed integer programming model (enhanced index tracking) for portfolio construction to track FBMKLCI Index in Malaysia. The optimization models are solved by using LINGO software [14].

\subsection{Index Tracking Model}

An optimization model is a mathematical decision-making tool that aims to find the values of decision variables that optimize an objective function among the set of all values of the decision variables that satisfy the given constraints [15-16]. Index tracking is a portfolio management that aims to construct the optimal portfolio by using the optimization model in order to generate similar return with the benchmark index return without purchasing all the index components. This objective can be achieved by minimizing the tracking error [2-3]. A single-stage mixed integer programming model [2, 10] was introduced to minimize the tracking error for index tracking problem [17-18]. The single-stage mixed integer programming model for index tracking is formulated as follows:

$$
\text { Minimize } E=\sqrt{\frac{1}{T} \sum_{i=1}^{T}\left(R_{P t}-R_{I t}\right)^{2}}
$$

Subject to

$$
\begin{gathered}
\sum_{i=1}^{N} Z_{i}=K \\
Z_{i} \in\{0,1\} \\
L_{i} Z_{i} \leq x_{i} \leq U_{i} Z_{i}
\end{gathered}
$$

where $E$ is the tracking error, $T$ is the number of periods, $R_{P t}$ is the return of the optimal portfolio at time $t$ and $R_{I t}$ is the return of the benchmark index at time $t, x_{i}$ is the weight of stock $i$ in the optimal portfolio, $N$ is the total number of stocks, $K$ is the number of stocks selected to track the benchmark index return, $Z_{i}$ is the binary integer, $L_{i}$ and $U_{i}$ are the lower and upper bounds of the investment proportion respectively on stock $i$.

Equation (1) is the objective function of the model which minimizes the tracking error. Constraint (2) ensures that the number of stocks in the optimal portfolio is $K$. For constraint 
(3), $Z_{i}=$ lindicates the $i$ th stock is included in the optimal portfolio or otherwise $Z_{i}=0$. Constraint (4) shows that the value of $x_{i}$ is limited in the interval $\left[L_{i}, U_{i}\right]$. Constraint (5) ensures that the total weight of stocks invested is one. Constraint (6) ensures that weight of the stock in the optimal portfolio is positive.

\subsection{Enhanced Index Tracking Model}

Enhanced index tracking is a portfolio management which aims to generate higher portfolio mean return than the benchmark index return besides minimizing the tracking error $[10,19]$. In this study, a two-stage approach is adopted in enhanced index tracking by improving the existing single-stage model. The first stage aims to minimize the tracking error as introduced in the existing model $[2,18]$. The second stage is proposed to maximize the portfolio mean return at minimum tracking error. The proposed two-stage mixed integer programming model for enhanced index tracking is formulated as follows:

First stage:

$$
\text { Minimize } E=\sqrt{\frac{1}{T} \sum_{i=1}^{T}\left(R_{P t}-R_{I t}\right)^{2}}
$$

Subject to

$$
\begin{gathered}
\sum_{i=1}^{N} Z_{i}=K \\
Z_{i} \in\{0,1\} \\
L_{i} Z_{i} \leq x_{i} \leq U_{i} Z_{i} \\
\sum_{i=1}^{N} x_{i}=1 \\
x_{i} \geq 0
\end{gathered}
$$

Second stage:

$$
\operatorname{Maximize} r_{P}=\sum_{i=1}^{N} r_{i} x_{i}
$$

Subject to

$$
\begin{aligned}
& \sum_{i=1}^{N} Z_{i}= \\
& Z_{i} \in\{0,1\}
\end{aligned}
$$




$$
\begin{gathered}
L_{i} Z_{i} \leq x_{i} \leq U_{i} Z_{i} \\
\sum_{i=1}^{N} x_{i}=1 \\
E^{*}-\delta \leq E \leq E^{*}+\delta \\
x_{i} \geq 0
\end{gathered}
$$

where $E$ is the tracking error, $T$ is the number of periods, $r_{P}$ is the mean return of the optimal portfolio, $r_{i}$ is the mean return of stock $i$ in the optimal portfolio, $R_{P t}$ is the return of the optimal portfolio at time $t, R_{I t}$ is the return of the benchmark index at time $t, R_{i t}$ is the return of stock $i$ at time $t, x_{i}$ is the weight of stock $i$ in the optimal portfolio, $N$ is the total number of stocks, $K$ is the number of stocks selected to track the benchmark index return, $Z_{i}$ is the binary integer, $L_{i}$ and $U_{i}$ are the lower and upper bounds of the fund proportion respectively on stock $i, E^{*}$ is the optimal value of tracking error which is determined in the first stage, $\delta$ is the allowable tolerance for tracking error.

Equation (7) is the objective function of the first stage model that minimizes the tracking error. Equation (13) is the objective function of the second stage model that maximizes the mean return of the optimal portfolio. Constraint (8) and (14) ensure that the number of stocks in the optimal portfolio is $K$. For constraint (9) and (15), $Z_{i}=1$ indicates the $i$ th stock is included in the optimal portfolio or otherwise $Z_{i}=0$. Constraint (10) and (16) show that the value of $x_{i}$ is limited in the interval $\left[L_{i}, U_{i}\right]$. Constraint (11) and (17) ensure that the total weight of stocks invested is one. Constraint (12) and (19) ensure that weight of the stock in the optimal portfolio is positive. For the second stage model, constraint (18) ensures that the tracking error is within the allowable tolerance based on the optimal value determined in the first stage model. In this study, the parameters are set as $K=10, \delta=0.0001, L_{i}=0.001$ and $U_{i}=1.00[10,18]$.

\subsection{Portfolio Performance}

Tracking error and mean return of the optimal portfolio are two elements in enhanced index tracking problem [11-12]. Tracking error is the standard deviation of the difference between the returns of the portfolio and the returns of the benchmark index [20-21]. The formula for tracking error is as follows. 


$$
T E=\sqrt{\frac{1}{T} \sum_{i=1}^{T}\left(R_{P t}-R_{I t}\right)^{2}}
$$

where $T E$ is the tracking error, $T$ is the number of periods, $R_{P t}$ is the return of the optimal portfolio at time $t, R_{I t}$ is the return of the benchmark index at time $t$.

The mean return of the portfolio is formulated as follows:

$$
r_{P}=\sum_{i=1}^{N} r_{i} x_{i}
$$

where $r_{P}$ is the mean return of the optimal portfolio, $x_{i}$ is the weight of stock $i$ in the optimal portfolio, $r_{i}$ is the mean return of stock $i$ in the optimal portfolio.

Excess return is defined as the difference between the portfolio mean return and benchmark index mean return which is formulated as follows [11, 22].

$$
E R=r_{P}-r_{I}
$$

where $E R$ is the excess return, $r_{P}$ is the mean return of the optimal portfolio, $r_{I}$ is the mean return of the benchmark index.

The performance of the optimal portfolio is measured with information ratio [23-24]. The information ratio is defined as the ratio of portfolio's excess mean return to the tracking error which is formulated as follows.

$$
I R=\frac{E R}{T E}
$$

where $I R$ is the information ratio, $E R$ is the excess mean return of the optimal portfolio over the mean return of the benchmark index, $T E$ is the tracking error. Higher information ratio indicates higher performance of the optimal portfolio.

\section{RESULTS AND DISCUSSION}

Table 1 presents the stock selection in optimal portfolio by both index tracking and enhanced index tracking models to track FBMKLCI Index. 
Table 1. Stock selection in optimal portfolio

\begin{tabular}{|c|c|c|}
\hline Stock & $\begin{array}{c}\text { Weights (\%) } \\
\text { Index Tracking Model } \\
\text { (Single Stage) }\end{array}$ & $\begin{array}{c}\text { Weights (\%) } \\
\text { Enhanced Index Tracking } \\
\text { Model (Two-Stage) }\end{array}$ \\
\hline AMMB & 0.00 & 0.00 \\
\hline AXIATA & 12.10 & 12.47 \\
\hline BAT & 0.00 & 0.00 \\
\hline CIMB & 8.69 & 8.86 \\
\hline DIGI & 10.41 & 8.79 \\
\hline GENTING & 8.72 & 8.75 \\
\hline GENM & 0.00 & 0.00 \\
\hline HLBANK & 0.00 & 0.00 \\
\hline HLFG & 0.00 & 0.00 \\
\hline IOICORP & 0.00 & 0.00 \\
\hline KLK & 0.00 & 0.00 \\
\hline MAYBANK & 12.63 & 7.92 \\
\hline MAXIS & 0.00 & 0.00 \\
\hline PETDAG & 0.00 & 4.77 \\
\hline PETGAS & 0.00 & 0.00 \\
\hline PBBANK & 16.79 & 20.76 \\
\hline PPB & 4.30 & 0.00 \\
\hline RHBCAP & 0.00 & 0.00 \\
\hline SIME & 12.43 & 10.59 \\
\hline $\mathrm{TM}$ & 0.00 & 0.00 \\
\hline TENAGA & 10.29 & 13.06 \\
\hline UMW & 0.00 & 0.00 \\
\hline YTL & 3.64 & 4.03 \\
\hline
\end{tabular}

As shown in Table 1, the list of stocks with positive weights indicates that those stocks are selected by the models in constructing the optimal portfolio to track FBMKLCI Index in 
Malaysia. The optimal portfolios for both models consist of 10 stocks with different weights in tracking FBMKLCI Index.

Fig. 1 and Fig. 2 present the optimal portfolio composition for both index tracking model and enhanced index tracking model respectively.

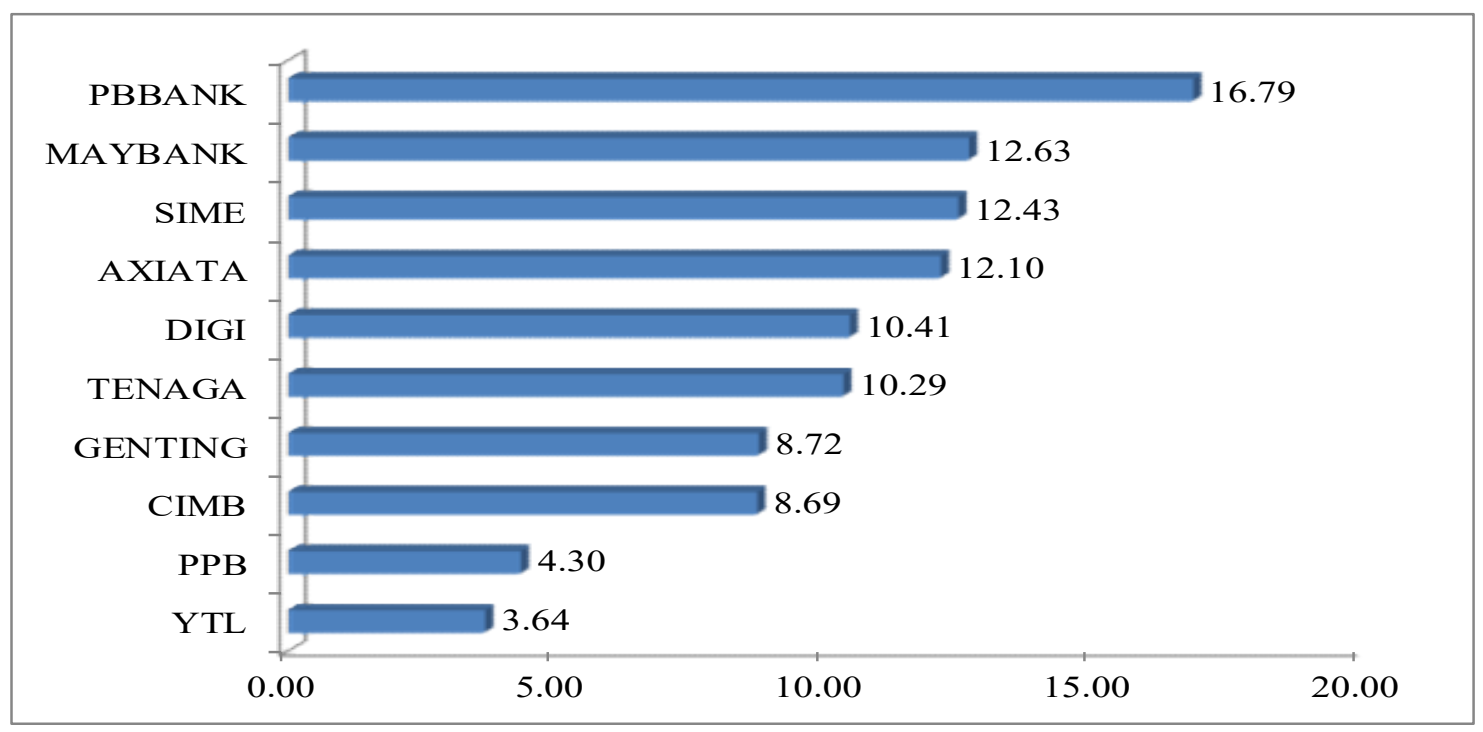

Fig.1.Optimal portfolio composition for index tracking model

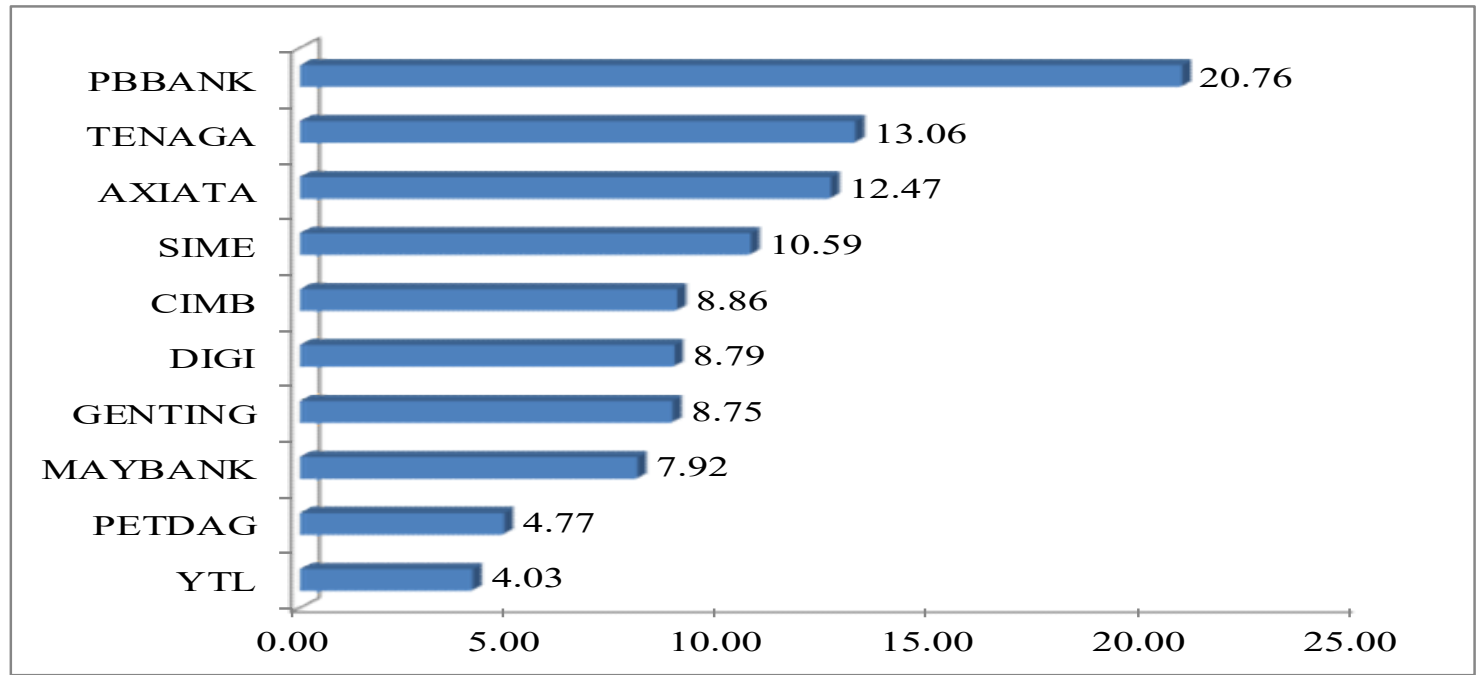

Fig.2. Optimal portfolio composition for enhanced index tracking model

Based on Fig. 2, the optimal portfolio for enhanced index tracking model consists of PBBANK (20.76\%), TENAGA (13.06\%), AXIATA (12.47\%), SIME (10.59\%), CIMB (8.86\%), DIGI (8.79\%), GENTING (8.75\%), MAYBANK (7.92\%), PETDAG (4.77\%) and YTL (4.03\%). This optimal portfolio composition is the optimal solution of the proposed enhanced index tracking model. 
As shown in both Fig. 1 and Fig. 2, both index tracking model and enhanced index tracking model give different weights of stock in the optimal portfolio. PBBANK is the most dominant stock in the optimal portfolios for both models. On the other hand, YTL is the smallest stock in the optimal portfolios for both models. Table 2 displays the optimal portfolio performance for enhanced index tracking model.

Table 2. Optimal portfolio performance for enhanced index tracking model

\begin{tabular}{ccc}
\hline Portfolio & FBMKLCI Index & Enhanced Index Tracking Model \\
\hline Number of stocks & 30 & 10 \\
Mean return (\%) & 0.1974 & 0.2591 \\
Excess return (\%) & - & 0.0617 \\
Tracking error (\%) & - & 0.1751 \\
Information ratio & - & 0.3524 \\
\hline
\end{tabular}

As shown in Table 2, the weekly mean return for FBMKLCI Index is $0.1974 \%$ based on the study period. The optimal portfolio for enhanced index tracking model tracks FBMKLCI Index with weekly mean return $0.2591 \%$ which is higher than the mean return of the index. This implies that the optimal portfolio for enhanced index tracking model is able to outperform FBMKLCI Index with weekly excess mean return $0.0617 \%$ at minimum tracking error $0.1751 \%$.

Table 3 presents the comparison of optimal portfolio performance between index tracking model and enhanced index tracking model.

Table 3. Comparison of optimal portfolio performance between index tracking model and enhanced index tracking model

\begin{tabular}{ccc}
\hline Portfolio & $\begin{array}{c}\text { Index Tracking } \\
\text { Model (Single Stage) }\end{array}$ & $\begin{array}{c}\text { Enhanced Index Tracking } \\
\text { Model (Two-Stage) }\end{array}$ \\
\hline Mean return (\%) & 0.2070 & 0.2591 \\
Excess return (\%) & 0.0096 & 0.0617 \\
Tracking error (\%) & 0.1451 & 0.1751 \\
Information ratio & 0.0662 & 0.3524 \\
\hline
\end{tabular}

As shown in Table 3, the enhanced index tracking model $(0.2591 \%)$ gives higher portfolio mean return than the index tracking model $(0.2070 \%)$. Therefore, the information ratio (ratio 
of excess return to tracking error) for the enhanced index tracking model (0.3524) is higher than the index tracking model $(0.0662)$. This implies that the proposed enhanced index tracking model is able to outperform the existing index tracking model in tracking FBMKLCI Index in Malaysia.

\section{CONCLUSION}

This paper proposes a two-stage mixed integer programming (enhanced index tracking) model to improve the existing single-stage mixed integer programming (index tracking) model. The proposed enhanced index tracking model is able to generate higher portfolio mean return than the benchmark FBMKLCI index in Malaysia. Besides that, the enhanced index tracking model is able to outperform the existing index tracking model with higher information ratio. The significance of this study is to propose a two-stage mixed integer programming model for enhanced index tracking which contributes $25 \%$ improvement on the portfolio mean return as compared to the existing single-stage model.

\section{ACKNOWLEDGEMENTS}

This study is supported by Universiti Kebangsaan Malaysia (UKM) research fund.

\section{REFERENCES}

[1] Lam W S, Lam W H. Strategic decision making in portfolio management with goal programming model. American Journal of Operations Management and Information Systems, 2016, 1(1): $34-38$

[2] Roll R. A mean variance analysis of tracking error. Journal of Portfolio Management, 1992, $18: 13-22$

[3] Meade N, Salkin G R. Developing and maintaining an equity index fund. Journal of Operation Research, 1990, 41(7):599-607

[4] Alexander C, Dimitriu A. Indexing and statistical arbitrage. Journal of Portfolio Management, 2005, 31(2):50-63

[5] Canakgoz N A, Beasley J E. Mixed integer programming approaches for index tracking and enhanced indexation. European Journal of Operational Research, 2008, 196:384-399 
[6] Guastaroba G, Speranza M G. Kernel Search: An application to index tracking problem. European Journal of Operational Research, 2012, 217:54-68

[7] Mezali H, Beasley J E. Quantile regression for index tracking and enhanced indexation. Journal of the Operational Research Society, 2013, 64:1676-1692

[8] Lam W S, Lam W H. Portfolio optimization for index tracking problem with mixed integer programming model. Journal of Scientific Research and Development, 2015, 2(10):5-8

[9] Lam W S, Saiful H J, Hamizun I. An empirical study on the characteristics of high risk aversion behavior in portfolio decision making using regression model. Advances in Environmental Biology, 2015, 9(7):17-20

[10] Beasley J E, Meade N, Chang T J. An evolutionary heuristics for the index tracking problem. European Journal of Operational Research, 2003, 148:621-643

[11] Lam W S, Lam W H. Mathematical modeling in enhanced index tracking with optimization model. Journal of Numerical Analysis and Applied Mathematics, 2016, 1:1-5

[12] Lam W S, Saiful H J, Hamizun I. Portfolio optimization for index tracking modelling in Malaysia stock market. AIP Conference Proceedings, 2016, 1739:1-5

[13] Lam W S, Saiful H J, Hamizun I. Enhanced index tracking modeling in portfolio optimization. AIP Conference Proceedings, 2013, 1557:469-472

[14] Lam W S, Saiful H J, Hamizun I. The impact of different economic scenarios towards portfolio selection in enhanced index tracking problem. Advanced Science Letters, 2015, 21(5):1285-1288

[15]Taha H. A. Operations research: An introduction.New Jersey: Pearson, 2017

[16] Winston W. L., Goldberg J. B. Operations research: Applications and algorithms.California: Thomson Learning, 2004

[17] Maringer D, Oyewumi O. Index tracking with constrained portfolios. Intelligent Systems in Accounting, Finance and Management, 2007, 15:57-71

[18] Lam W S, Saiful H J, Hamizun I. Comparison between two stage regression model and variance model in portfolio optimization. Journal of Applied Science and Agriculture, 2014, $9(18): 36-40$

[19] Lam W S, Saiful H J, Hamizun I. The impact of human behavior towards portfolio 
selection in Malaysia. Procedia of Social and Behavioral Sciences, 2015, 172:674-678

[20] Lam W S, Saiful H J, Hamizun I. Index tracking modeling in portfolio optimization with mixed integer linear programming. Journal of Applied Science and Agriculture, 2014, $9(18): 47-50$

[21] Lam W S, Saiful H J, Hamizun I. An empirical comparison of different optimization models in enhanced index tracking problem. Advanced Science Letters, 2015, 21(5):1278-1281

[22] Wu L C, Chou S C, Yang C C, Ong C S. Enhanced index investing based on goal programming. Journal of Portfolio Management, 2007, 33:49-56

[23] Wu L C, Wu L H. Tracking a benchmark index-using a spreadsheet-based decision support system as the driver. Expert Systems, 2012, 30:79-88

[24] Israelsen C L. A refinement to the sharpe ratio and information ratio. Journal of Asset Management, 2005, 5(6):423-427

\section{How to cite this article:}

Lam, W. S, Saiful, H. J.and Lam, W. H. Enhanced index tracking in portfolio optimization with two-stage mixed integer programming model. J. Fundam. Appl. Sci., 2017, 9(5S), 1-12. 